\title{
Structural Basis for the Structure-Activity Relationships of Peroxisome Proliferator-Activated Receptor Agonists
}

Neeraj Mahindroo, ${ }^{\dagger}$ Yi-Hui Peng, ${ }^{\dagger, \dagger}$ Chia-Hui Lin, ${ }^{\S}$ Uan-Kang Tan, ${ }^{\text {Il }}$ Ekambaranellore Prakash, ${ }^{\dagger}$ Tzu-

Wen Lien, ${ }^{\dagger}$ I-Lin Lu, ${ }^{\dagger, \diamond}$ Hong-Jen Lee, ${ }^{\dagger}$ John Tsu-An Hsu, ${ }^{\dagger, \#}$ Xin Chen ${ }^{\dagger}$ Chun-Chen Liao ${ }^{\S}$ Ping-Chiang Lyu ${ }^{\ddagger}$ Yu-Sheng Chao, ${ }^{\dagger}$ Su-Ying $W u,{ }^{\dagger, *}$ and Hsing-Pang Hsieh ${ }^{\dagger}, *$

Division of Biotechnology and Pharmaceutical Research, National Health Research Institutes, 35,

Keyan Road, Zhunan Town, Miaoli County 350, Taiwan, Republic of China. Department of Chemistry, Department of Life Sciences, and Department of Chemical Engineering, National Tsing Hua University, Hsinchu 300, Taiwan, Republic of China. Graduate Institute of Life Sciences, National Defense Medical Center, Taipei 114, Taiwan, Republic of China. Department of Chemical Engineering, Northern Taiwan Institute of Science and Technology, Taipei 112, Taiwan, Republic of China.

\section{Contents:}

1. Chemical Methods

2. Table1. ${ }^{1}$ H NMR, HRMS, HPLC Purity Data for Compounds 7-12.

3. Cell Culture and PPAR Transactivation Assay (TA).

4. Structural Biology Studies.

5. Table 2. Interaction analysis of PPAR $\gamma \mathrm{LBD}$ protein amino acids with the ligands.

\footnotetext{
${ }^{\dagger}$ National Health Research Institutes.

* Department of Life Sciences, National Tsing Hua University.

${ }^{\S}$ Department of Chemistry, National Tsing Hua University.

"I Department of Chemical Engineering, Northern Taiwan Institute of Science and Technology

${ }^{\diamond}$ Graduate Institute of Life Sciences, National Defense Medical Center.

\# Department of Chemical Engineering, National Tsing Hua University.

* To whom correspondence should be addressed. S.-Y. Wu: phone: 886-37-246-166 ext. 35713; fax: 886-37-586-456; e-mail: suying@ @hri.org.tw; H.-P. Hsieh: phone: 886-37-246-166 ext. 35708; fax: 886-37-586-456; e-mail: hphsieh@nhri.org.tw.
} 
Chemical Methods. Nuclear magnetic resonance $\left({ }^{1} \mathrm{H}\right.$ NMR) spectra were obtained with a Varian Mercury-300 spectrometer operating at $300 \mathrm{MHz}$ with chemical shift in parts per million (ppm, $\delta$ ) downfield from TMS as an internal standard. High-resolution mass spectra (HRMS) were measured with a Finnigan (MAT-95XL) electron impact (EI) and fast atom bombardment (FAB) mass spectrometer. Analytical purity was assessed by RP-HPLC using an Agilent (Hewlett Packard) 1100 series system equipped with a diode array spectrometer. Flash column chromatography was done using silica gel (Merck Kieselgel 60, No. 9385, 230-400 mesh ASTM). Reactions were monitored by TLC using Merck $60 \mathrm{~F}_{254}$ silica gel glass backed plates $(5 \times 10 \mathrm{~cm})$; zones were detected visually under ultraviolet irradiation $(254 \mathrm{~nm})$ or by spraying with phosphomolybdic acid reagent (Aldrich) followed by heating at $80^{\circ} \mathrm{C}$. All solvents were dried according to standard procedures. All reagents were used as purchased without further treatment unless otherwise stated. All reactions were carried out under an atmosphere of dry nitrogen.

Table 1. ${ }^{1} \mathrm{H}$ NMR, HRMS and HPLC Purity Data for Compounds 7-12.

\begin{tabular}{|c|c|c|c|c|}
\hline \multirow{2}{*}{ Compound } & \multirow{2}{*}{${ }^{1} \mathrm{H}$ NMR } & \multirow{2}{*}{ HRMS } & \multicolumn{2}{|c|}{ HPLC Purity } \\
\hline & & & Solvent $\mathrm{A}^{a}$ & Solvent $\mathrm{B}^{b}$ \\
\hline 7 & $\begin{array}{l}\left(300 \mathrm{MHz}, \mathrm{CDCl}_{3}\right) \delta 1.03(\mathrm{t}, J=7.5 \\
\mathrm{Hz}, 3 \mathrm{H}), 1.59-1.71(\mathrm{~m}, 2 \mathrm{H}), 2.26 \\
\text { (quintet, } J=6.0 \mathrm{~Hz}, 2 \mathrm{H}), 3.08(\mathrm{t}, J= \\
7.5 \mathrm{~Hz}, 2 \mathrm{H}), 3.94(\mathrm{t}, J=5.4 \mathrm{~Hz}, 2 \mathrm{H}), \\
4.33(\mathrm{t}, J=6.3 \mathrm{~Hz}, 2 \mathrm{H}), 4.66(\mathrm{~s}, 2 \mathrm{H}), \\
6.34-6.37(\mathrm{~m}, 1 \mathrm{H}), 6.55(\mathrm{~d}, J=3.0 \mathrm{~Hz}, \\
1 \mathrm{H}), 6.93-6.99(\mathrm{~m}, 3 \mathrm{H}), 7.07(\mathrm{~d}, J= \\
9.0 \mathrm{~Hz}, 1 \mathrm{H}), 7.39-7.44(\mathrm{~m}, 2 \mathrm{H}), 7.50- \\
7.55(\mathrm{~m}, 1 \mathrm{H}), 7.65(\mathrm{~d}, J=9.0 \mathrm{~Hz}, 1 \mathrm{H}), \\
7.74-7.77(\mathrm{~m}, 2 \mathrm{H}), 7.87(\mathrm{dd}, J=1.5, \\
9.0 \mathrm{~Hz}, 1 \mathrm{H}), 7.97(\mathrm{~d}, J=9.0 \mathrm{~Hz}, 1 \mathrm{H}) \\
8.12(\mathrm{~d}, J=1.5 \mathrm{~Hz}, 1 \mathrm{H})\end{array}$ & $\begin{array}{l}\text { HRMS }\left(\mathrm{EI}^{+}\right. \\
m / z) \text { calcd for } \\
\mathrm{C}_{33} \mathrm{H}_{31} \mathrm{O}_{5} \mathrm{~N}_{1} \\
521.2202 \text {, found } \\
521.2202 \text {. }\end{array}$ & 95.1029 & 96.7355 \\
\hline 8 & $\left(300 \mathrm{MHz}, \mathrm{CDCl}_{3}\right) \delta 1.03(\mathrm{t}, J=7.5$ & HRMS $\left(\mathrm{EI}^{+}\right.$ & 97.4943 & 96.7495 \\
\hline
\end{tabular}




\begin{tabular}{|c|c|c|c|c|}
\hline & $\begin{array}{l}\mathrm{Hz}, 3 \mathrm{H}), 1.63-1.71(\mathrm{~m}, 2 \mathrm{H}), 2.27 \\
\text { (quintet, } J=6.0 \mathrm{~Hz}, 2 \mathrm{H}), 3.09(\mathrm{t}, J= \\
7.5 \mathrm{~Hz}, 2 \mathrm{H}), 3.96(\mathrm{t}, J=5.4 \mathrm{~Hz}, 2 \mathrm{H}), \\
4.33(\mathrm{t}, J=6.6 \mathrm{~Hz}, 2 \mathrm{H}), 4.62(\mathrm{~s}, 2 \mathrm{H}), \\
6.39(\mathrm{dd}, J=0.9,3.0 \mathrm{~Hz}, 1 \mathrm{H}), 6.83 \\
(\mathrm{dd}, J=2.7,9.0 \mathrm{~Hz}, 1 \mathrm{H}), 7.00-7.02 \\
(\mathrm{~m}, 2 \mathrm{H}), 7.08(\mathrm{~d}, J=9.0 \mathrm{~Hz}, 1 \mathrm{H}), 7.20 \\
(\mathrm{~d}, J=9.0 \mathrm{~Hz}, 1 \mathrm{H}), 7.39-7.45(\mathrm{~m}, 2 \mathrm{H}), \\
7.49-7.55(\mathrm{~m}, 1 \mathrm{H}), 7.66(\mathrm{~d}, J=9.0 \mathrm{~Hz}, \\
1 \mathrm{H}), 7.75-7.78(\mathrm{~m}, 2 \mathrm{H}), 7.88(\mathrm{dd}, J= \\
1.8,9.0 \mathrm{~Hz}, 1 \mathrm{H}), 7.98(\mathrm{~d}, J=9.0 \mathrm{~Hz}, \\
1 \mathrm{H}) 8.12(\mathrm{~d}, J=1.8 \mathrm{~Hz}, 1 \mathrm{H})\end{array}$ & $\begin{array}{l}m / z) \text { calcd for } \\
\mathrm{C}_{33} \mathrm{H}_{31} \mathrm{O}_{5} \mathrm{~N}_{1} \\
521.2202 \text {, found } \\
521.2206 \text {. }\end{array}$ & & \\
\hline 9 & $\begin{array}{l}\left(300 \mathrm{MHz}, \mathrm{CDCl}_{3}\right) \delta 1.06(\mathrm{t}, J=7.5 \\
\mathrm{Hz}, 3 \mathrm{H}), 1.65-1.74(\mathrm{~m}, 2 \mathrm{H}), 2.22 \\
\text { (quintet, } 2 \mathrm{H}), 3.12(\mathrm{t}, J=7.5 \mathrm{~Hz}, 2 \mathrm{H}), \\
3.87(\mathrm{t}, J=5.4 \mathrm{~Hz}, 2 \mathrm{H}), 4.26(\mathrm{t}, J= \\
6.3 \mathrm{~Hz}, 2 \mathrm{H}), 4.39(\mathrm{~s}, 2 \mathrm{H}), 6.36(\mathrm{~d}, J= \\
2.4 \mathrm{~Hz}, 1 \mathrm{H}), 6.78(\mathrm{dd}, J=2.1,8.4 \mathrm{~Hz}, \\
1 \mathrm{H}), 6.96(\mathrm{~d}, \mathrm{~J}=2.4 \mathrm{~Hz}, 1 \mathrm{H}), 7.10(\mathrm{~d}, \\
J=3.0 \mathrm{~Hz}, 1 \mathrm{H}), 7.19(\mathrm{~d}, J=8.7 \mathrm{~Hz}, \\
1 \mathrm{H}), 7.26-7.49(\mathrm{~m}, 3 \mathrm{H}), 7.56-7.63(\mathrm{~m}, \\
1 \mathrm{H}), 7.81(\mathrm{~d}, J=9.0 \mathrm{~Hz}, 1 \mathrm{H}), 7.96 \\
(\mathrm{dd}, J=1.2,8.3 \mathrm{~Hz}, 2 \mathrm{H}), 7.97(\mathrm{dd}, J= \\
1.8,8.7 \mathrm{~Hz}, 1 \mathrm{H}), 8.07(\mathrm{~d}, J=9.0 \mathrm{~Hz}, \\
1 \mathrm{H}), 8.14(\mathrm{~s}, 1 \mathrm{H})\end{array}$ & $\begin{array}{l}\text { HRMS }\left(\mathrm{EI}^{+}\right. \\
m / z) \text { calcd for } \\
\mathrm{C}_{33} \mathrm{H}_{31} \mathrm{O}_{5} \mathrm{~N}_{1} \\
521.2202 \text {, found }\end{array}$ & 96.0303 & 95.5059 \\
\hline 10 & $\begin{array}{l}\left(300 \mathrm{MHz}, \mathrm{CDCl}_{3}\right) \delta 1.12(\mathrm{t}, J=7.5 \\
\mathrm{Hz}, 3 \mathrm{H}), 1.67(\mathrm{~s}, 6 \mathrm{H}), 1.71-1.80(\mathrm{~m}, \\
2 \mathrm{H}), 2.36 \quad \text { (quintet, } J=6.0 \mathrm{~Hz}, 2 \mathrm{H}), \\
3.18(\mathrm{t}, J=7.5 \mathrm{~Hz}, 2 \mathrm{H}), 4.07(\mathrm{t}, J= \\
5.4 \mathrm{~Hz}, 2 \mathrm{H}), 4.44(\mathrm{t}, J=6.6 \mathrm{~Hz}, 2 \mathrm{H}), \\
6.54(\mathrm{~d}, J=3.0 \mathrm{~Hz}, 1 \mathrm{H}), 6.61(\mathrm{dd}, J= \\
0.6,7.5 \mathrm{~Hz}, 1 \mathrm{H}), 7.05(\mathrm{~d}, J=3.0 \mathrm{~Hz}, \\
1 \mathrm{H}), 7.07-7.20(\mathrm{~m}, 4 \mathrm{H}), 7.49-7.62(\mathrm{~m},\end{array}$ & $\begin{array}{l}\text { HRMS }\left(\mathrm{EI}^{+}\right. \\
m / z) \text { calcd for } \\
\mathrm{C}_{35} \mathrm{H}_{35} \mathrm{O}_{5} \mathrm{~N} \\
549.2515, \text { found } \\
549.2508\end{array}$ & 96.7981 & 97.1959 \\
\hline
\end{tabular}




\begin{tabular}{|c|c|c|c|c|}
\hline & $\begin{array}{l}4 \mathrm{H}), 7.61(\mathrm{~d}, J=8.7 \mathrm{~Hz}, 1 \mathrm{H}), 7.88- \\
7.84(\mathrm{~m}, 2 \mathrm{H}), 7.97(\mathrm{dd}, J=1.8,9.0 \\
\mathrm{Hz}, 1 \mathrm{H}), 8.07(\mathrm{~d}, J=9.0 \mathrm{~Hz}, 1 \mathrm{H}), 8.22 \\
(\mathrm{~d}, J=1.8 \mathrm{~Hz}, 1 \mathrm{H})\end{array}$ & & & \\
\hline 11 & $\begin{array}{l}\left(300 \mathrm{MHz}, \mathrm{CDCl}_{3}\right) \delta 1.11(\mathrm{t}, J=7.5 \\
\mathrm{Hz}, 3 \mathrm{H}), 1.56(\mathrm{~s}, 6 \mathrm{H}), 1.72-1.79(\mathrm{~m}, \\
2 \mathrm{H}), 2.37 \text { (quintet, } J=6.0 \mathrm{~Hz}, 2 \mathrm{H}), \\
3.17(\mathrm{t}, J=7.8 \mathrm{~Hz}, 2 \mathrm{H}), 4.07(\mathrm{t}, J= \\
6.0 \mathrm{~Hz}, 2 \mathrm{H}), 4.43(\mathrm{t}, J=6.0 \mathrm{~Hz}, 2 \mathrm{H}), \\
6.44(\mathrm{~d}, J=3.0 \mathrm{~Hz}, 1 \mathrm{H}), 6.85(\mathrm{dd}, J= \\
2.4,9.0 \mathrm{~Hz}, 1 \mathrm{H}), 7.12(\mathrm{~d}, J=2.7 \mathrm{~Hz}, \\
1 \mathrm{H}), 7.18(\mathrm{~d}, J=9.0 \mathrm{~Hz}, 1 \mathrm{H}), 7.24(\mathrm{~d}, \\
J=2.1 \mathrm{~Hz}, 1 \mathrm{H}), 7.261(\mathrm{~d}, J=9.0 \mathrm{~Hz}, \\
1 \mathrm{H}), 7.48-7.54(\mathrm{~m}, 2 \mathrm{H}), 7.58-7.64(\mathrm{~m}, \\
1 \mathrm{H}), 7.75(\mathrm{~d}, J=9.0 \mathrm{~Hz}, 1 \mathrm{H}), 7.84- \\
7.87(\mathrm{~m}, 2 \mathrm{H}), 7.97(\mathrm{dd}, J=1.8,9.0 \\
\mathrm{Hz}, 1 \mathrm{H}), 8.07(\mathrm{~d}, J=9.0 \mathrm{~Hz}, 1 \mathrm{H}), 8.22 \\
(\mathrm{~d}, J=1.5 \mathrm{~Hz}, 1 \mathrm{H}) .\end{array}$ & $\begin{array}{l}\text { HRMS }\left(\mathrm{EI}^{+}\right. \\
m / z) \text { calcd for } \\
\mathrm{C}_{35} \mathrm{H}_{35} \mathrm{O}_{5} \mathrm{~N} \\
549.2515 \text {, found } \\
549.2499 \text {. }\end{array}$ & 97.5468 & 96.1049 \\
\hline 12 & $\begin{array}{l}\left(300 \mathrm{MHz}, \mathrm{CDCl}_{3}\right) \delta 1.11(\mathrm{t}, J=7.5 \\
\mathrm{Hz}, 3 \mathrm{H}), 1.53(\mathrm{~s}, 6 \mathrm{H}), 1.53-1.77(\mathrm{~m}, \\
2 \mathrm{H}), 2.34 \text { (quintet, } J=6.0 \mathrm{~Hz}, 2 \mathrm{H}), \\
3.18(\mathrm{t}, J=7.5 \mathrm{~Hz}, 2 \mathrm{H}), 4.05(\mathrm{t}, J= \\
5.7 \mathrm{~Hz}, 2 \mathrm{H}), 4.39(\mathrm{t}, J=6.3 \mathrm{~Hz}, 2 \mathrm{H}), \\
6.46(\mathrm{~d}, J=2.4 \mathrm{~Hz}, 1 \mathrm{H}), 6.79(\mathrm{dd}, J= \\
2.1,8.4 \mathrm{~Hz}, 1 \mathrm{H}), 6.99(\mathrm{~d}, J=2.4 \mathrm{~Hz}, \\
1 \mathrm{H}), 7.08(\mathrm{~d}, J=3.0 \mathrm{~Hz}, 1 \mathrm{H}), 7.17(\mathrm{~d}, \\
J=8.7 \mathrm{~Hz}, 1 \mathrm{H}), 7.54-7.49(\mathrm{~m}, 3 \mathrm{H}), \\
7.62-7.59(\mathrm{~m}, 1 \mathrm{H}), 7.75(\mathrm{~d}, J=9.0 \mathrm{~Hz}, \\
1 \mathrm{H}), 7.85(\mathrm{dd}, J=1.2,8.3 \mathrm{~Hz}, 2 \mathrm{H}), \\
7.97(\mathrm{dd}, J=1.8,8.7 \mathrm{~Hz}, 1 \mathrm{H}), 8.07(\mathrm{~d}, \\
\mathrm{J}=9.0 \mathrm{~Hz}, 1 \mathrm{H}), 8.21(\mathrm{~d}, J=1.8 \mathrm{~Hz}, \\
1 \mathrm{H})\end{array}$ & $\begin{array}{l}\text { HRMS } \text { EI }^{+} \\
m / z) \text { calcd for } \\
\mathrm{C}_{35} \mathrm{H}_{35} \mathrm{O}_{5} \mathrm{~N} \\
549.2515 \text {, found } \\
549.2501\end{array}$ & 95.0375 & 95.4059 \\
\hline
\end{tabular}

${ }^{a}$ Solvent System A: Acetonitrile and $10 \mathrm{mM}$ Sodium acetate/ 0.1\% Formic Acid /Water - Gradient (10\%-90\%). Column - ZORBAX Eclipse XDB-C8 $3 \times 150 \mathrm{~mm} 5 \mu \mathrm{M} .{ }^{b}$ Solvent System B :. 
Acetonitrile and 0.1\% H3PO4/H2O - Gradient (10\%-90\%). Column - ZORBAX Eclipse XDB-C8 4.6 $\times 150 \mathrm{~mm} 5 \mu \mathrm{M}$.

Cell Culture and PPAR Transactivation Assay (TA). Activities of compounds were evaluated for functional activity in a cell-based transcription assays using Gal-4-PPAR chimeric receptors as described previously. ${ }^{1}$ Huh-7 cells were seeded at $1 \times 10^{5}$ cells/well in 24 -well cell culture plates in Dulbecco's modified Eagle's medium (DMEM) containing 10\% fetal bovine serum (Gemini BioProducts), 100 units/ml penicillin $\mathrm{G}$, and $100 \mathrm{mg} / \mathrm{ml}$ streptomycin sulfate and cells were maintained at $37^{\circ} \mathrm{C}$ in a humidified atmosphere of $5 \% \mathrm{CO}_{2}$. After $24 \mathrm{~h}$, transfections were performed with transfection reagent (Roche, Indianapolis, IN, 11988 387) according to the instructions provided by the manufacturer. Briefly, transfection mixtures for each well contained $0.48 \mu \mathrm{l}$ of transfection reagent, 40 ng of pcDNA3-GAL4/PPAR expression vector, $137 \mathrm{ng}$ of pUAS(5x)-tk-luc reporter vector, and $0.245 \mathrm{ng}$ of SV40-Ren as an internal control for transfection efficiency. Cells were incubated in the transfection mixture for $6 \mathrm{~h}$ at $37{ }^{\circ} \mathrm{C}$ in an atmosphere of $5 \% \mathrm{CO}_{2}$. The cells were then incubated for another $24 \mathrm{~h}$ in fresh culture medium with various concentrations of test compounds. Since the compound stock solutions were prepared in $\mathrm{Me}_{2} \mathrm{SO}$, control cells were incubated with equivalent concentrations of $\mathrm{Me}_{2} \mathrm{SO}$. Final $\mathrm{Me}_{2} \mathrm{SO}$ concentration up to $0.1 \%$ was shown not to affect the cell-based transactivation activity. Cell lysates were produced using Reporter Lysis Buffer (Promega, Madison, WI) according to the manufacturer's instructions. Luciferase activity in cell extracts was determined using Luciferase Assay kit (Promega, Madison, WI) in a SIRIUS-0 luminometer (Berthold detection systems, Pforzheim, Germany).

\section{Structural Biology Studies}

Expression and purification of PPAR $\gamma$-LBD (PPAR $\gamma$ ligand binding domain). PPAR $\gamma \mathrm{LBD}$, starting from amino acid 207 to 477, was cloned with glutathione $S$-transferase (GST) in the pGEX-6P-1 vector. The recombinant plasmid with GST-PPAR $\gamma$ LBD gene were transformed into BL21 (DE3) $E$. coli cells and grown at $37{ }^{\circ} \mathrm{C}$ for $2 \mathrm{~h}$ to reach $\mathrm{OD}_{600}=0.6$. Following the addition of isopropyl- $\beta$-Dthiogalactopytanoside, the culture was incubated for another $6 \mathrm{~h}$ at $22{ }^{\circ} \mathrm{C}$. Cells were then harvested by 
centrifugation and resuspended in lysis buffer $(50 \mathrm{mM}$ Tris- $\mathrm{Cl}(\mathrm{pH} 7.5), 1 \mathrm{mM}$ EDTA and $0.1 \mathrm{mM}$ PMSF, buffer A). After adding lysozyme to disrupt the cell membrane, the lysate was centrifuged at $19000 \mathrm{rpm}$ for $30 \mathrm{~min}$ at $4{ }^{\circ} \mathrm{C}$. The supernatant was then loaded onto Q-sepharose ion exchanger column (Hiprep 16/10 Q XL, Amersham Biosciences), the bound protein was eluted with a liner gradient of $0 \%$ to $100 \%$ of buffer $\mathrm{B}$ (buffer $\mathrm{A}+300 \mathrm{mM} \mathrm{NaCl}$ ). The fraction containing GSTPPAR $\gamma$ LBD were further purified by GST affinity column (GSTPrep FF 16/10, Amersham Biosciences). The column was first washed with PBS buffer to remove the unbound proteins and subsequently eluted by Tris buffer (50mM tris $\mathrm{pH} 7.5)$ with $10 \mathrm{mM}$ GSH buffer to obtain the GST fusion protein. The eluate was then digested with PreScission protease (Amersham Biosciences) overnight at $4{ }^{\circ} \mathrm{C}$. The GST tag was removed by passage through the GST affinity column (GSTPrep FF 16/10, Amersham Biosciences). The PPAR $\gamma \mathrm{LBD}$ was concentrated and exchanged to the buffer consisting of $20 \mathrm{mM}$ Tris- $\mathrm{Cl}$ (pH 8.0), 5 mM DTT, $100 \mathrm{mM} \mathrm{NaCl}$ and $0.5 \mathrm{mM}$ EDTA. The purity of PPAR $\gamma L B D$ was checked by SDS-PAGE gels before crystallization.

Crystallization and Structure Determination. Typically, $10 \mu \mathrm{L}$ PPAR $\gamma \mathrm{LBD}(8.0 \mathrm{mg} / \mathrm{ml}$ in the buffer of $20 \mathrm{mM}$ Tris-Cl (pH 8.0), $5 \mathrm{mM}$ DTT, $100 \mathrm{mM} \mathrm{NaCl}$ and $0.5 \mathrm{mM}$ EDTA) was mixed with 0.5 $\mu \mathrm{L}$ of compound (10 mM in a buffer of $20 \mathrm{mM}$ Tris- $\mathrm{Cl}(\mathrm{pH} 8.0), 5 \mathrm{mM}$ DTT, $100 \mathrm{mM} \mathrm{NaCl}$ and 0.5 mM EDTA) and equilibrated for $1 \mathrm{~h}$ on ice. For crystallization, $1.5 \mu \mathrm{L}$ complex solution was added to $1.5 \mu \mathrm{L}$ of well solution containing 28.0 28.5\% PEG3350. After 3-7 days at $18{ }^{\circ} \mathrm{C}$, the complex crystals of an average size of $0.2 \mathrm{~mm}$ were obtained.

The crystal was soaked in a cryoprotectant containing 0-3\% glycerol and then flash-frozen in liquid nitrogen. Diffraction data were collected at the NSRRC on station BL13B1 and BL13C1. The data were processed by $\mathrm{DENZO}^{2}$ and reduced with SCALEPACK. The structure was solved by molecular replacement by MOLREP $^{3}$ using a monomer of published PPAR $\gamma$ LBD structure (PDB code 2PRG) as the search model. The program REFMAC ${ }^{4}$ and SHELX ${ }^{5}$ were used for structural refinement and the model building were carried out with the program O. ${ }^{6}$ All the figures were draw by PyMOL. ${ }^{7}$ 
Table 2. Interaction analysis of PPAR $\gamma \mathrm{LBD}$ protein amino acids with the ligands.
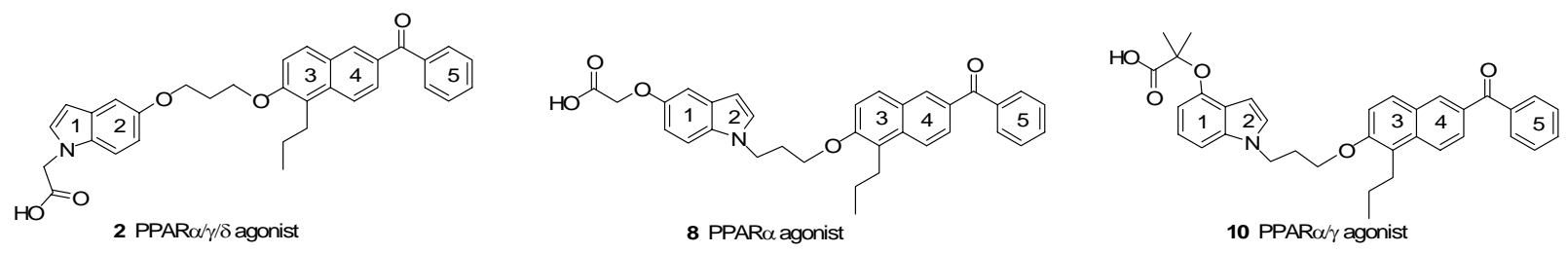

\begin{tabular}{|c|c|c|c|}
\hline Compd & 2 & 8 & 10 \\
\hline $\mathrm{COOH}$ & $\begin{array}{l}\mathrm{H} 449 \\
\mathrm{Y} 473\end{array}$ & - & $\begin{array}{ll}\text { S289、H323 } \\
\text { H449、Y } 473\end{array}$ \\
\hline di- $\mathrm{CH}_{3}$ group & - & - & F282、C285、Q286 \\
\hline Ring1 & $\begin{array}{c}\text { F282、C285、 } \\
\text { H449 }\end{array}$ & $\begin{array}{r}\text { F282、C285、 } \\
\text { F363、H449 }\end{array}$ & $\begin{array}{l}\text { F282、C285、 } \\
\text { F363、M364、H449 }\end{array}$ \\
\hline Ring2 & Y327、M364 & Y327、K367 & L330 \\
\hline linker & $\begin{array}{l}\text { L330、V339 } \\
\text { M364、K367 }\end{array}$ & F363、M364 & L330、K367 \\
\hline Ring3 & $\begin{array}{c}\text { C285、I341 } \\
\text { M348 }\end{array}$ & I281、C 285 & I341 \\
\hline Ring4 & G284、I341 & G284、C285 & $\mathrm{G} 284$ \\
\hline Ring5 & $\begin{array}{r}\text { L255、E259、 } \\
\text { I341、M348 }\end{array}$ & G284 & L255、E259 \\
\hline Propyl group & $\mathrm{C} 285$ & $\mathrm{R} 288$ & C285、L330 \\
\hline
\end{tabular}

\section{References:}

(1) Mahindroo, N.; Huang, C. F.; Peng, Y. H.; Wang, C. C.; Liao, C. C.; Lien, T. W.; Chittimalla, S. K.; Huang, W. J.; Chai, C. H.; Prakash, E.; Chen, C. P.; Hsu, T. A.; Peng, C. H.; Lu, I. L.; Lee, L. H.; Chang, Y. W.; Chen, W. C.; Chou, Y. C.; Chen, C. T.; Goparaju, C. M. V.; Chen, Y. S.; Lan, S. J.; Yu, M. C.; Chen, X.; Chao, Y. S.; Wu, S. Y.; Hsieh, H. P. Novel Indole-Based Peroxisome Proliferator-Activated Receptor Agonists: Design, SAR, Structural Biology, and Biological Activities. J. Med. Chem. 2005, 48, 8194-8208.

(2) Otwinowski, Z.; Minor, W. Processing of X-ray Diffraction Data Collected in Oscillation Mode. Methods in Enzymology 1997, 276, 307-326.

(3) Vagin, A.; Teplyakov, A. MOLREP: An Automated Program for Molecular Replacement. J. Appl. Cryst. 1997, 30, 1022-1025. 
(4) Murshudov, G. N.; Vagin, A. A.; Dodson, E. J. Refinement of Macromolecular Structures by the Maximum-likelihood Method. Acta Cryst. 1997, D53, 240-255.

(5) Sheldrick, G. M.; Schneider, T. R. SHELXL: High-Resolution Refinement. Methods in Enzymlolgy 1997, 277, 319-343.

(6) Jones, T. A.; Zou, J. Y.; Cowan, S. W.; Kjeldgaard, M. Improved Methods for Building Protein Models in Electron Density Maps and the Location of Errors in these Models. Acta Cryst. 1991, A47, 110-119.

(7) DeLano, W. L. The PyMOL Molecular Graphics System. 2002, DeLano Scientific, San Carlos, CA, USA. 